\title{
Low-mass di-muon searches with the LHCb detector
}

\section{Langenbruch ${ }^{* \dagger}$}

I. Physikalisches Institut, RWTH Aachen University, Aachen, Germany

E-mail: christoph. langenbruchecern.ch

Muon pairs of opposite charge allow for powerful searches for phenomena beyond the Standard Model. The LHCb experiment is particularly well suited for the analysis of di-muons at low invariant masses due to its flexible trigger system, excellent vertex locator, invariant mass resolution and forward acceptance. These proceedings present recent results from $\mathrm{LHCb}$ on searches for new exotic particles and measurements of rare decays with di-muon final states.

The European Physical Society Conference on High Energy Physics

5-12 July, 2017

Venice

* Speaker.

${ }^{\dagger}$ On behalf of the LHCb collaboration 


\section{Introduction}

Many extensions of the Standard Model (SM) predict the existence of particles decaying to a muon pair of opposite charge. Examples of these New Physics models include dark sector theories that predict the existence of a new hidden sector of particles that only interact feebly with the Standard Model (for a recent review see Ref. [1]). Furthermore, rare decays of neutral mesons to di-muon final states provide powerful tests of the Standard Model.

The LHCb experiment is ideally suited for the search for di-muon final states in the $\mathrm{GeV}$ mass range due to its forward geometry with a pseudorapidity coverage of $2<\eta<5$ and its precise tracking system that provides excellent mass resolution. In addition, the LHCb muon system exhibits high muon identification efficiencies of $97 \%$ at low misidentification probabilities of $1-3 \%$. Finally, the flexible LHCb trigger system, consisting of a first level trigger (L0) implemented in hardware and a second high level trigger stage (HLT) implemented in software, provides very low $p_{\mathrm{T}}(\mu)$ thresholds $\left(p_{\mathrm{T}}(\mu) \gtrsim 1.8 \mathrm{GeV}\right.$ at L0) and thus high trigger efficiencies. Since the start of Run 2 the HLT performs a full online calibration and alignment, which allows the use of particle identification variables in the trigger algorithms. For Run 3 the trigger will be fully implemented in software which will further increase the trigger efficiency for low-mass di-muon events.

\section{Searches for hidden sector bosons}

Rare $b \rightarrow s \mu^{+} \mu^{-}$penguin decays are an excellent place to search for hidden sector particles $\chi$ mixing with the Higgs sector as they are dominated by the contribution from the heavy top quark. The LHCb experiment has performed searches for the decays $B^{0} \rightarrow K^{* 0} \chi\left(\rightarrow \mu^{+} \mu^{-}\right)$[2] and $B^{+} \rightarrow K^{+} \chi\left(\rightarrow \mu^{+} \mu^{-}\right)$[3] using data corresponding to an integrated luminosity of $3 \mathrm{fb}^{-1}$ taken during the LHC Run 1. Backgrounds are suppressed by using a boosted decision tree and the reconstructed $B$ mass as discriminants. The di-muon invariant mass is scanned for prompt and detached di-muon vertices (depending on the lifetime and the lifetime significance of the di-muon system) to search for an excess of $\chi$ signal candidates. Regions with narrow SM resonances with dimuon final states (e.g. the $\phi, J / \psi, \psi(2 S)$, etc.) are vetoed. No excesses are found and upper limits on the braching fractions of $B^{0} \rightarrow K^{* 0} \chi\left(\rightarrow \mu^{+} \mu^{-}\right)$and $B^{+} \rightarrow K^{+} \chi\left(\rightarrow \mu^{+} \mu^{-}\right)$are set depending on the lifetime and the invariant mass of the di-muon system as shown in Fig. 1.
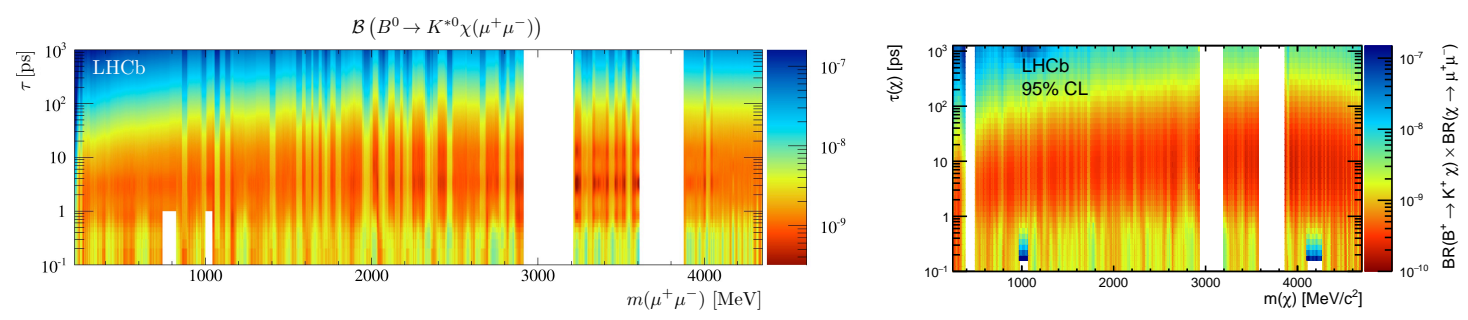

Figure 1: Upper limits at 95\% CL, depending on the lifetime and mass of the hidden sector boson $\chi$, for (left) the decay $B^{0} \rightarrow K^{* 0} \chi\left(\rightarrow \mu^{+} \mu^{-}\right)$[2] and (right) the decay $B^{+} \rightarrow K^{+} \chi\left(\rightarrow \mu^{+} \mu^{-}\right)$[3].

These upper limits can be translated to exclusion limits for specific models. Figure 2 gives the resulting exclusion limits for the axion model detailed in Ref. [4] and for the inflaton model from 
Ref. [5]. The exclusion limits for the inflaton model given depend on the $\chi$ invariant mass and the mixing angle $\theta$, and show that the LHCb measurements can exclude most of the parameter space below $\sim 4 \mathrm{GeV}$.
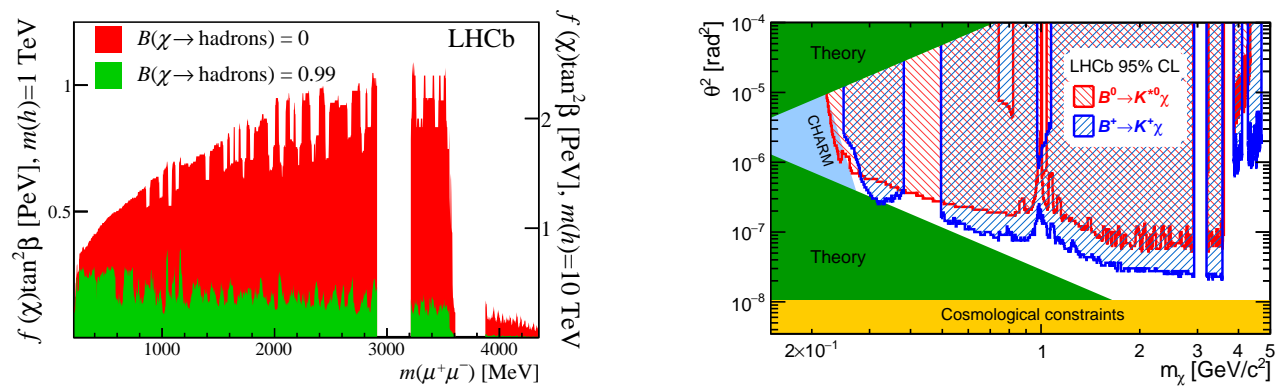

Figure 2: (Left) exclusion limits for the axion model in Ref. [4] for large $\tan \beta$ and charged Higgs masses at 95\% CL [2]. (Right) constraints on the mixing angle $\theta$ between Higgs and inflaton fields for the inflaton model detailed in Ref. [5] at 95\% CL [3].

\section{Dark photon searches}

An attractive possibility for models in which dark forces mediate between the dark sector and SM particles is the existence of dark photons $A^{\prime}$ that couple to the SM photon via kinetic mixing with strength $\varepsilon \sqrt{\alpha_{\mathrm{EM}}}$. A search for the decays dark photons $A^{\prime} \rightarrow \mu^{+} \mu^{-}$has been performed with the $\mathrm{LHCb}$ experiment using a data sample corresponding to an integrated luminosity of $1.6 \mathrm{fb}^{-1}$ taken during Run 2 [12]. The search includes prompt and long-lived $A^{\prime}$ decays. For prompt decays the search is performed from near the di-muon threshold to $70 \mathrm{GeV}$, the long-lived search is performed in the mass range $214<m\left(A^{\prime}\right)<350 \mathrm{MeV}$.

Figure 3 gives preliminary exclusion limits at $90 \% \mathrm{CL}$ depending on $m\left(A^{\prime}\right)$ and $\varepsilon^{2}$. For the prompt search the limits are comparable to the best existing limits below $0.5 \mathrm{GeV}$ and the most stringent limits for the mass range $10.6<m\left(A^{\prime}\right)<70 \mathrm{GeV}$. The limits on long-lived dark photons are the first obtained using a detached vertex signature.
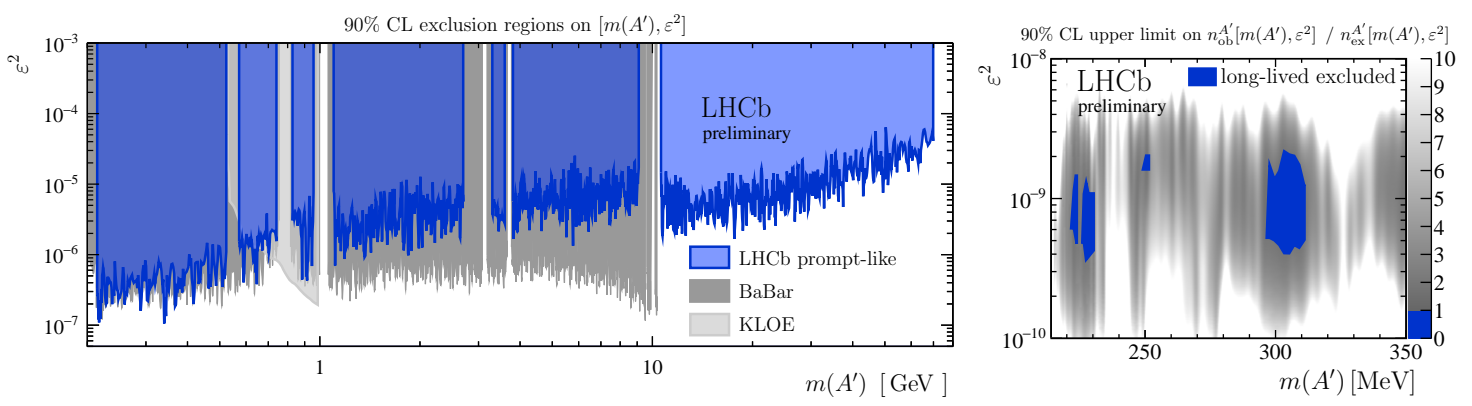

Figure 3: Preliminary exclusion limits at $90 \% \mathrm{CL}$ for the search for (left) prompt $A^{\prime} \rightarrow \mu^{+} \mu^{-}$decays and (right) long-lived $A^{\prime} \rightarrow \mu^{+} \mu^{-}$decays [12]. For the prompt search exclusion limits from BaBar [13] and KLOE [14] are given as well.

The removal of the hardware trigger and increase in luminosity in Run 3 will lead to a potential increase in the number of expected $A^{\prime} \rightarrow \mu^{+} \mu^{-}$decays by a factor of $\mathscr{O}(100-1000)$, significantly 
extending the sensitivity of the LHCb experiment to dark photons. Figure 4 from Ref. [6] gives the prospects for the search for $A^{\prime}$ decays with an integrated luminosity of $15 \mathrm{fb}^{-1}$ at LHCb. Extrapolations for the search for $A^{\prime} \rightarrow \mu^{+} \mu^{-}$are given in red, with expected limits from a search for $A^{\prime} \rightarrow e^{+} e^{-}$in blue. The search for the di-electron final state was proposed in Ref. [15] using $D^{* 0} \rightarrow D^{0} \gamma\left(\rightarrow A^{\prime} \rightarrow e^{+} e^{-}\right)$decays.

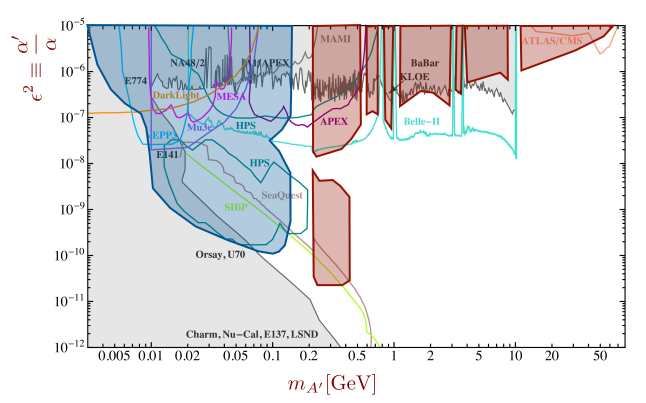

Figure 4: Expected exclusion limits for dark photon searches depending on $m\left(A^{\prime}\right)$ and $\varepsilon^{2}$ from (red shaded) a planned LHCb search for $A^{\prime} \rightarrow \mu^{+} \mu^{-}$and (blue shaded) a planned LHCb search for $A^{\prime} \rightarrow e^{+} e^{-}$in comparison to current and future limits from other experiments [6].

\section{The rare decays $B_{s}^{0} \rightarrow \mu^{+} \mu^{-}$and $B^{0} \rightarrow \mu^{+} \mu^{-}$}

The decays $B_{s}^{0} \rightarrow \mu^{+} \mu^{-}$and $B^{0} \rightarrow \mu^{+} \mu^{-}$proceed via flavour changing neutral currents and are thus loop-suppressed in the SM. Furthermore, the decays are helicity suppressed resulting in very low predictions for their branching fractions in the SM of $\mathscr{B}\left(B_{s}^{0} \rightarrow \mu^{+} \mu^{-}\right)=(3.65 \pm 0.23) \times 10^{-9}$ and $\mathscr{B}\left(B^{0} \rightarrow \mu^{+} \mu^{-}\right)=(1.06 \pm 0.09) \times 10^{-10}$ [7]. Compared to the $B_{s}^{0}$ decay the $B^{0}$ mode is further suppressed by the ratio of CKM matrix elements $\left|V_{\mathrm{td}} / V_{\mathrm{ts}}\right|^{2}$ in the SM. These decays are particularly sensitive probes for new scalar and pseudoscalar contributions.

The LHCb experiment has performed an analysis of $B_{(s)}^{0} \rightarrow \mu^{+} \mu^{-}$using $3 \mathrm{fb}^{-1}$ of data taken during Run 1 and $1.4 \mathrm{fb}^{-1}$ of data taken during Run 2 [8]. LHCb is able to perform the first observation of the very rare decay $B_{s}^{0} \rightarrow \mu^{+} \mu^{-}$by a single experiment with a significance of $7.8 \sigma$. The signal candidates in the most sensitive region are shown in Fig. 5. No evidence for $B^{0} \rightarrow \mu^{+} \mu^{-}$ is observed, the significance of this mode is determined to be $1.6 \sigma$ and an upper limit of $\mathscr{B}\left(B^{0} \rightarrow\right.$ $\left.\mu^{+} \mu^{-}\right)<3.4 \times 10^{-10}$ is set at $95 \% \mathrm{CL}$. The branching fractions of the two decays are measured to be

$$
\begin{aligned}
& \mathscr{B}\left(B_{s}^{0} \rightarrow \mu^{+} \mu^{-}\right)=\left(3.0 \pm 0.6_{-0.2}^{+0.3}\right) \times 10^{-9} \\
& \mathscr{B}\left(B^{0} \rightarrow \mu^{+} \mu^{-}\right)=\left(1.5_{-1.0}^{+1.2}{ }_{-0.1}^{+0.2}\right) \times 10^{-10}
\end{aligned}
$$

as shown in Fig. 5. The branching fractions are in good agreement with the SM prediction.

Furthermore, the first measurement of the effective lifetime $\tau_{\mu \mu}$ is performed for the decay $B_{s}^{0} \rightarrow \mu^{+} \mu^{-}$. This observable gives orthogonal information to the branching fraction and would allow to distinguish between scalar and non-scalar New Physics contributions [9]. The effective lifetime is given by

$$
\tau_{\mu \mu}=\frac{\tau_{B_{s}^{0}}}{1-y_{s}^{2}}\left[\frac{1+2 A_{\Delta \Gamma}^{\mu \mu} y_{s}+y_{s}^{2}}{1+A_{\Delta \Gamma}^{\mu \mu} y_{s}}\right]
$$



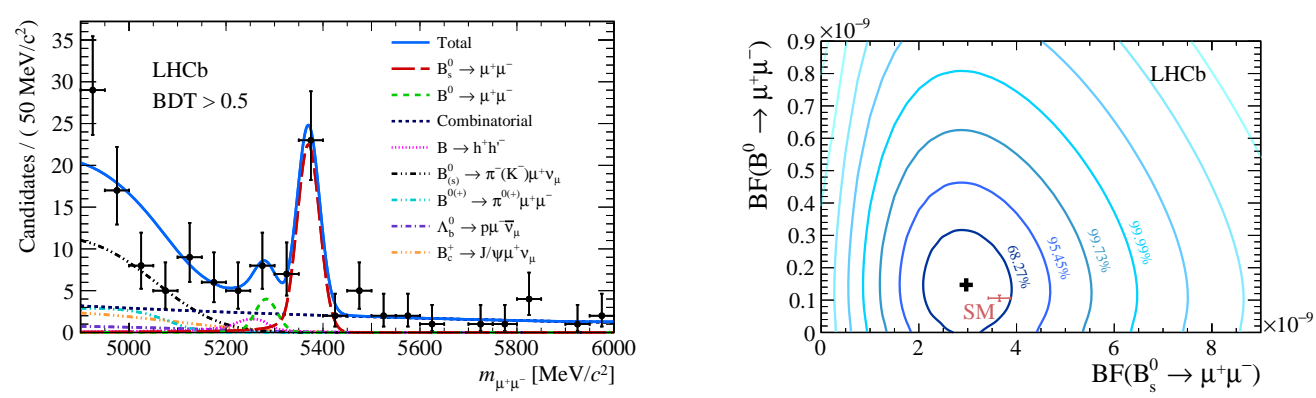

Figure 5: (Left) signal-like candidates with the fit for the yields of $B_{s}^{0} \rightarrow \mu^{+} \mu^{-}$and $B^{0} \rightarrow \mu^{+} \mu^{-}$overlaid and (right) two-dimensional confidence regions for the branching fractions of the two modes [8].

where $\tau_{B_{s}^{0}}=1.510 \pm 0.005 \mathrm{ps}$ is the $B_{s}^{0}$ mean lifetime and $y_{s}=\tau_{B_{s}^{0}} \Delta \Gamma / 2=0.062 \pm 0.006[10,11]$. The parameter $A_{\Delta \Gamma}^{\mu \mu}$ is equal to +1 in the SM, but can have any value in the range $[-1,+1]$ in NP models. LHCb performs the first measurement of the effective lifetime and finds $\tau_{\mu \mu}=2.04 \pm$ $0.44 \pm 0.05 \mathrm{ps}$, in agreement with the SM at $1.0 \sigma$.

\section{Conclusions}

Recent results from the $\mathrm{LHCb}$ experiment on searches for exotic di-muon resonances and analyses of rare decays with di-muon final states have been presented. Exclusion limits have been set that are able to significantly constrain the parameter space of many models of New Physics. Future improvements in the LHCb trigger system in Run 3 will further increase the sensitivity to low-mass di-muon decays.

\section{References}

[1] J. Alexander et al., arXiv:1608.08632 [hep-ph].

[2] R. Aaij et al. [LHCb Collaboration], PRL 115 (2015) no.16, 161802 [arXiv:1508.04094 [hep-ex]].

[3] R. Aaij et al. [LHCb Collaboration], PRD 95 (2017) no.7, 071101 [arXiv:1612.07818 [hep-ex]].

[4] M. Freytsis et al., PRD 81 (2010) 034001 [arXiv:0911.5355 [hep-ph]].

[5] F. Bezrukov and D. Gorbunov, PLB 736 (2014) 494 [arXiv:1403.4638 [hep-ph]].

[6] P. Ilten et al., PRL 116 (2016) no.25, 251803 [arXiv:1603.08926 [hep-ph]].

[7] C. Bobeth et al., PRL 112 (2014) 101801 [arXiv:1311.0903 [hep-ph]].

[8] R. Aaij et al. [LHCb Collaboration], PRL 118 (2017) no.19, 191801 [arXiv:1703.05747 [hep-ex]].

[9] K. De Bruyn et al., PRL 109 (2012) 041801 [arXiv:1204.1737 [hep-ph]].

[10] Y. Amhis et al., arXiv:1612.07233 [hep-ex].

[11] C. Patrignani et al. [Particle Data Group], Chin. Phys. C 40 (2016) no.10, 100001.

[12] R. Aaij et al. [LHCb Collaboration], LHCb-PAPER-2017-038, To be submitted to PRL

[13] J. P. Lees et al. [BaBar Collaboration], PRL 113 (2014) no.20, 201801 [arXiv:1406.2980 [hep-ex]].

[14] A. Anastasi et al. [KLOE-2 Collaboration], PLB 757 (2016) 356 [arXiv:1603.06086 [hep-ex]].

[15] P. Ilten et al., PRD 92 (2015) no.11, 115017 [arXiv:1509.06765 [hep-ph]]. 